\title{
La prothèse maxillo-faciale au sein d'un centre de lutte contre le cancer
}

\section{PRÉFACE}

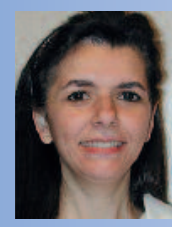

Ilham EL ACHHAB

Prothésiste maxillo-faciale et épithésiste Service de chirurgie cervico-faciale, odontologie.

Institut Gustave Roussy,

39 rue Camille Desmoulins 94800 Villejuif.
Nous ne sommes plus à l'époque des gueules cassées, des grands traumas non reconstruits ou des chirurgies carcinologiques très mutilantes dont l'exérèse complète était le seul but ; époque où la prothèse maxillo-faciale ne faisait que combler au mieux les vastes pertes de substance.

Aujourd'hui, les grands traumas sont le plus souvent réparés chirurgicalement ainsi que les malformations congénitales et notre spécialité s'inscrit presque essentiellement dans les réhabilitations dentaire et maxillo-faciale en rapport avec la chirurgie des tumeurs mais de façon bien différente.

En effet, avec les progrès de la chirurgie plastique et reconstructrice, il ne s'agit plus seulement de combler les pertes de substances en postopératoire, mais de réaliser des conformateurs, des patrons, des guides chirurgicaux, etc. en préopératoire pour tendre au résultat escompté et ensuite de concevoir et d'adapter des prothèses à des volumes nouveaux dans la cavité buccale et non à de simples pertes de substances.

Des étapes de chirurgie préprothétiques sont d'ailleurs souvent nécessaires avant l'appareillage définitif.

La réalisation de ces prothèses pré- et postopératoires immédiates ainsi qu'en final l'appareillage d'usage ne peut se faire qu'avec un prothésiste expérimenté, rompu à cette spécialité dont la collaboration étroite, sur place, avec le reste de l'équipe médicale, surtout le chirurgien et l'odontologue est indispensable pour l'obtention d'un résultat optimal de nos prothèses tant sur le plan fonctionnel qu'esthétique.

$$
\begin{gathered}
\text { Dr J.-P. MARGAINAUD } \\
\text { Chef du service } \\
\text { de chirurgie cervico-faciale, } \\
\text { odontologie } \\
\text { de l'Institut Gustave Roussy, } \\
94800 \text { Villejuif }
\end{gathered}
$$




\section{Introduction}

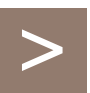

Les pertes de substances secondaires aux résections de cancer de l'extrémité céphalique posent des problèmes de réhabilitations complexes où l'approche multidisciplinaire entre chirurgiens ORL, plasticiens, odontologistes, radiothérapeutes, psychiatres, orthophonistes, prothésistes est impérative.

Le but est de proposer au malade le meilleur mariage de l'ensemble de ces disciplines. La participation du prothésiste est primordiale, car il permet de répondre à des problèmes complexes qu'aucune autre discipline n'est apte à prendre en charge $[6,7]$. Ainsi, sa contribution permettra de disposer de prothèses et d'épithèses pour de multiples situations cliniques, qui feront appel à chaque fois à son sens de la créativité, de l'esthétique et d'adaptation.

La réhabilitation maxillo-faciale est beaucoup plus complexe qu'une prothèse dentaire traditionnelle car il s'agit de travailler sur des pertes de substances qu'il faut imaginer et recréer tout en tenant compte du confort du patient, mais elle ne peut être menée à bien par les seuls chirurgiens, odontologues et prothésistes maxillofaciaux. D'autres spécialistes entrent en ligne de compte :

- l'orthophoniste ;

- le radiothérapeute ;

- le psychiatre.

L'orthophoniste et le radiothérapeute peuvent demander des aménagements spéciaux dans la conception de la prothèse. Afin de rééduquer l'ouverture buccale du patient, une mécanothérapie nous est demandée par l'orthophoniste... Le radiothérapeute nous indique le type de protection qu'il souhaite avoir afin de protéger les zones non irradiées. Le prothésiste qui a conçu et réalisé la prothèse en collaboration avec le praticien est lui aussi en contact étroit avec le patient. Tout au long de sa maladie, le patient sera revu régulièrement dans le service pour ajouter un élément, modifier ou remplacer l'appareillage. Souvent un lien affectif va se créer entre eux. La variété des activités ne peut être efficace que s'il y a une étroite collaboration entre le prothésiste, les chirurgiens dentistes, les chirurgiens ORL et plasticiens [3].

Lorsqu'une tumeur maligne est diagnostiquée, le plan de traitement est étudié et discuté par tous les membres de l'équipe médicale. Le chirurgien précise et prévoit les limites de résection, le prothésiste maxillo-facial et l'odontologue donnent des indications sur la prothèse qui va être réalisée et sur les points d'appui souhaitables pour fixer la prothèse envisagée.

Après que le chirurgien-dentiste ait pris une empreinte de la cavité buccale, le prothésiste maxillo-facial peut intervenir. Tout commence par le choix des matériaux. Ils doivent être le plus adaptés possible : résine, plâtre, cire, silicone et métaux... Ce choix se fait en concertation avec le praticien, le prothésiste et le patient. Le prothésiste va créer une maquette en moulant, modelant et sculptant la prothèse qui sera essayée par le dentiste ou le chirurgien ORL et éventuellement retouchée par le prothésiste afin de ne pas blesser ni gêner le patient.

Les prothèses, qu'elles soient maxillaires ou faciales, doivent répondre à des exigences fonctionnelles, esthétiques et anatomiques. Huit à dix heures de travail sont généralement nécessaires à la conception et à la fabrication d'une prothèse. Pour toutes ces raisons, elles sont très coûteuses. 


\section{Les différentes familles}

\section{de prothèses maxillo-faciales}

Il en existe de nombreuses sortes. Les plus courantes sont les suivantes.

\section{Les moyens de contention}

Ils sont utilisés dans le traitement des fractures. On y retrouve des ligatures, des appareils de blocage. Ils prennent appui sur des dents restantes. On distingue :

\section{> Les appareils de blocage}

Ils sont utilisés afin de conserver la bonne relation intermaxillaire lors de la greffe du péroné (fig 1 à 4).
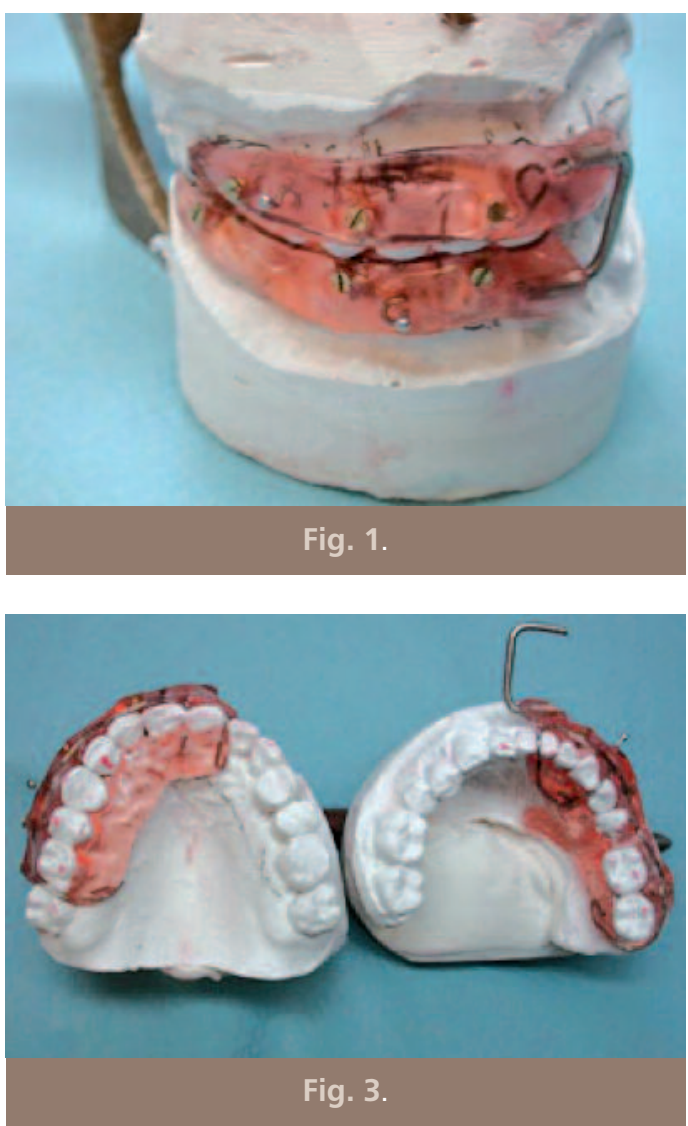

\section{> Les prothèses de reconstruction mandibulaire}

Elles sont destinées à assurer le remplacement de la mandibule et doivent être considérées comme des endoprothèses.

Elles peuvent se substituer complètement à l'os qu'elles remplacent sur une partie de sa longueur ou bien seconder temporairement un greffon osseux pendant sa période de consolidation.
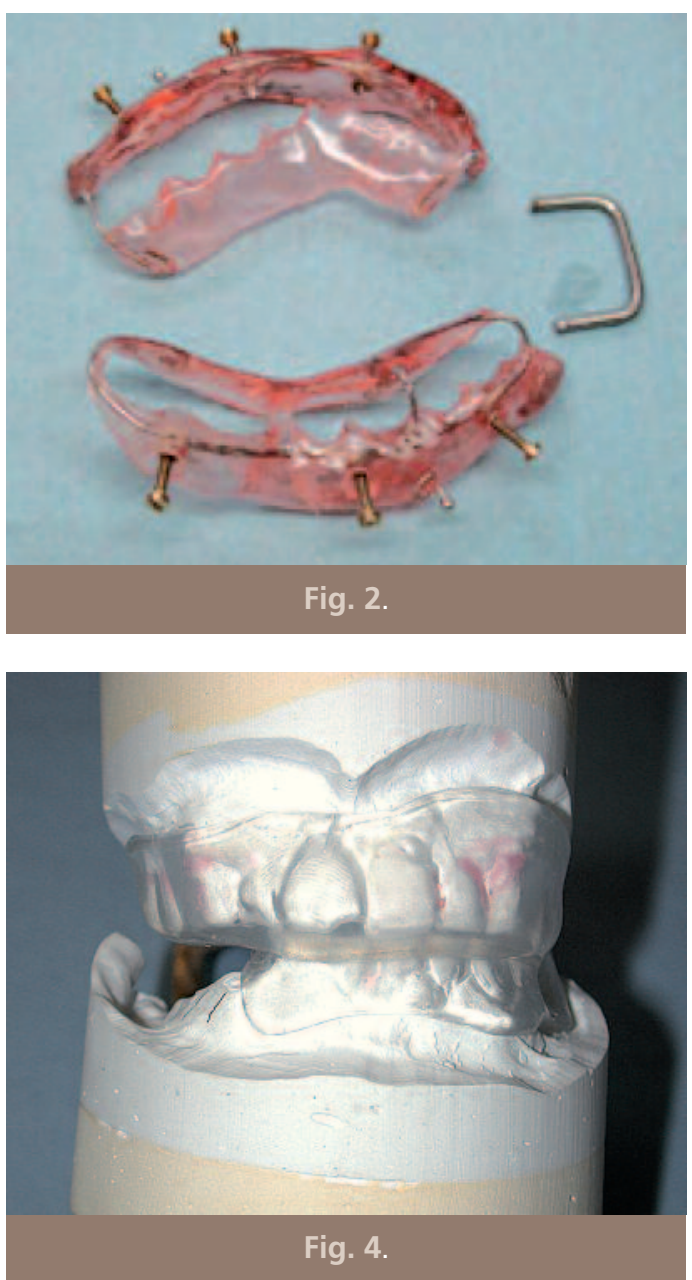


\section{Les moyens de correction et les conformateurs}

Ils sont utilisés dans le traitement chirurgical des tumeurs des maxillaires et nécessitent une étude préalable des corrections sur les moulages avant l'ostéotomie et le maintien de cette correction après I'intervention chirurgicale. Ces prothèses comportent également des appareils guides et des appareils mobilisateurs. On distingue :

\section{$>$ Les appareils guides}

Ils permettent de prévenir l'apparition d'une déviation tout en gardant une mobilité de la mandibule. Ils ont une double fonction :

- s'opposer aux rétractions fibreuses ;

- assurer en occlusion un articulé normal (fig 5).
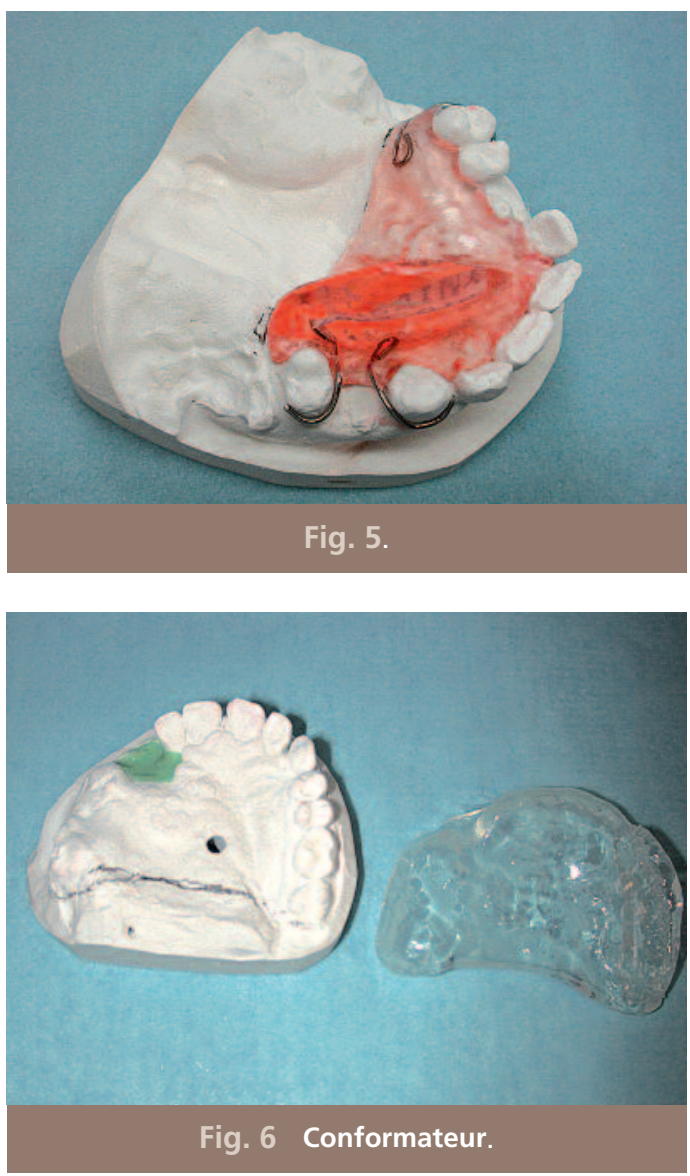

\section{$>$ Les conformateurs}

Ils sont utilisés pour guider la cicatrisation et sont destinés à maintenir des lambeaux muqueux ou cutanés. Ils sont préparés avant I'intervention (fig. 6 et 7). Les empreintes sont coulées en plâtre puis modifiées en fonction de I'intervention. On rectifie en fonction de la forme et des limites des contours osseux utilisables pour la sustentation de la prothèse. Celle-ci est réalisée en résine acrylique transparente, ce qui permet de contrôler la muqueuse sous-jacente. La prothèse conformatrice sera portée pendant le temps de la réalisation de la prothèse définitive mais aussi utilisée afin de protéger le lambeau du frottement de la langue. Afin de limiter le bourgeonnement des tissus greffés, celles-ci sont réalisées dans un premier temps avec des plaques souples thermoformées. L'avantage de ce matériau est la facilité d'emploi et d'être non traumatisant pour le patient. Ces conformateurs servent aussi à maintenir du tulle gras afin de limiter le bourgeonnement. Celui-ci sera modifié toutes les semaines qui suivent à la demande du chirurgien afin de réadapter le conformateur, jusqu'à obtenir le volume souhaité. Par la suite, une plaque avec des crochets est réalisée en résine acrylique transparente, afin de permettre au

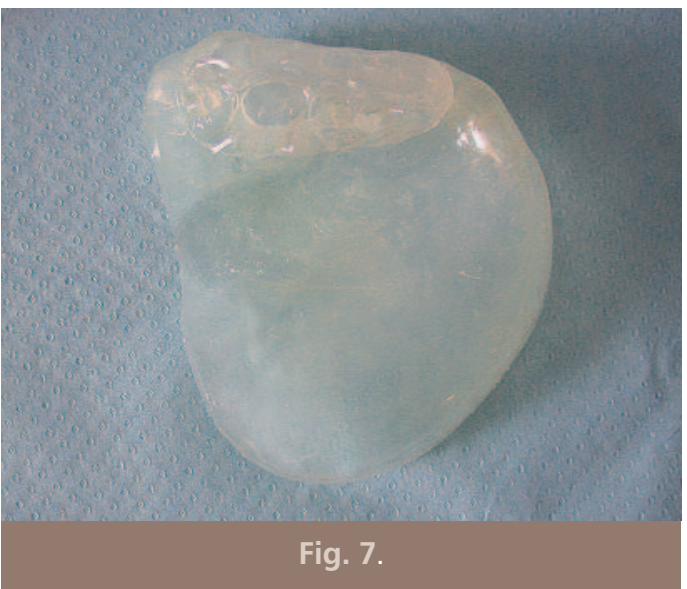


patient de s'alimenter. Le patient portera cette plaque jusqu'à cicatrisation

\section{$>$ Les conformateurs narinaires et oculaires}

Ce sont des « mainteneurs d'espaces " pour éviter brides cicatricielles et rétraction tissulaire. Ils
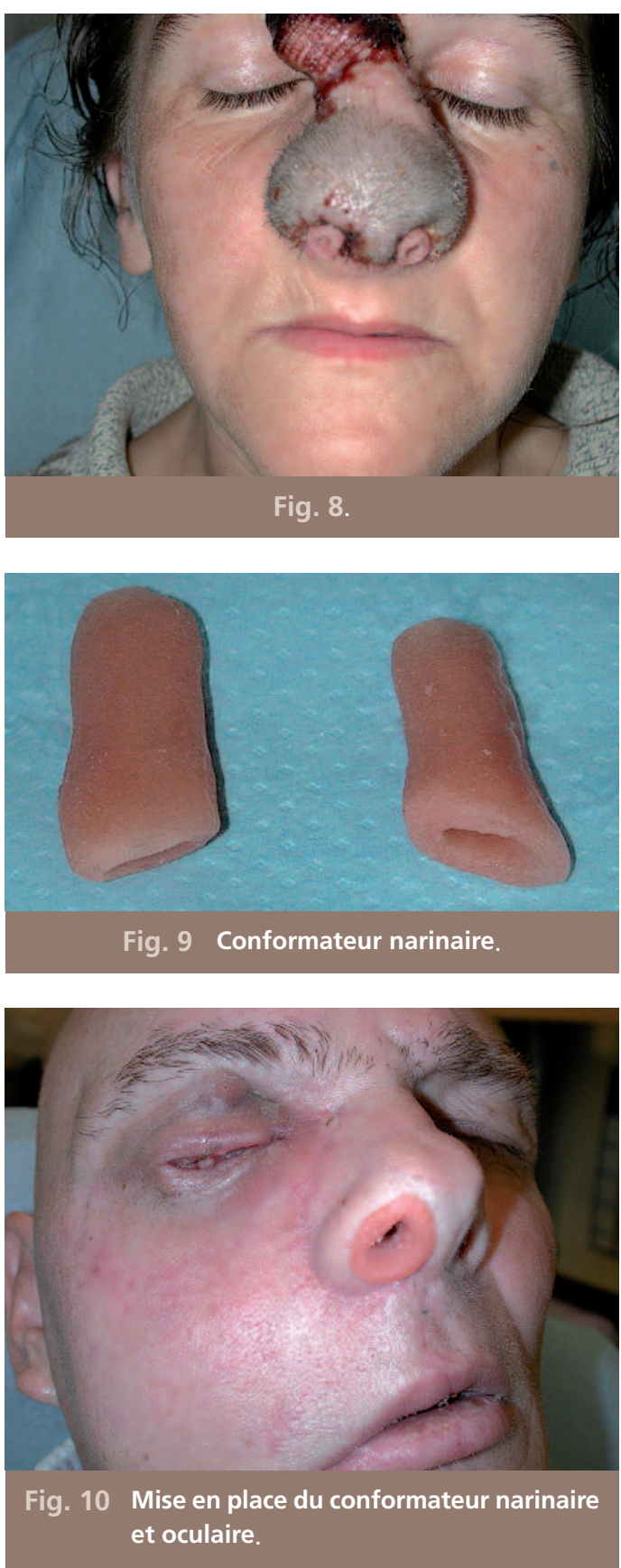

sont placés en peropératoire et laissés en place pendant tout le phénomène de cicatrisation. Une prothèse définitive vient les remplacer dans le cas de l'orbite (fig. 8 à 10).

\section{Les moyens de comblements}

\section{> Les prothèses vélopalatines}

Leurs fonctions sont de constituer un support à I'obturateur pharyngien lorsqu'il y a une fente palatine. Elles assurent son obturation et restituent la phonation tout en corrigeant l'articulé dentaire et en remplaçant les dents absentes.

Ces plaques sont toujours préparées en préopératoire lors de la consultation d'odontologie systématique. Après que le malade ait porté cette plaque pendant plusieurs jours et qu'elle ait été ajustée à plusieurs reprises, l'étape de confection de la partie obturatrice est commencée. Pour cela, le chirurgien-dentiste détermine l'état buccodentaire et la solidité des dents qui vont servir comme support de prothèse. L'empreinte est prise en alginate à l'aide d'un "porte-empreinte " individuel confectionné avec une résine photopolymérisable. À partir de cette empreinte et de son antagoniste mandibulaire, les modèles en plâtre dur sont coulés. L'enregistrement de l'occlusion et la mise en articulateur sont effectués. On détermine alors les moyens de sustentation de la plaque. La stabilité de cette dernière doit être très précise, surtout en arrière, car le poids de I'obturateur risque de décrocher la prothèse.

Les crochets sont un moyen de rétention pour la prothèse. Ceux-ci peuvent être coulés lorsqu'ils sont associés à un châssis métallique ou façonnés en fils d'acier et ajustés à la pince dans le cas d'une plaque en résine. Après essayage et ajustage, la prothèse est mise en moufle et transformée en résine acrylique. Le rebasage de l'obturateur sur la plaque est réalisé grâce à un 


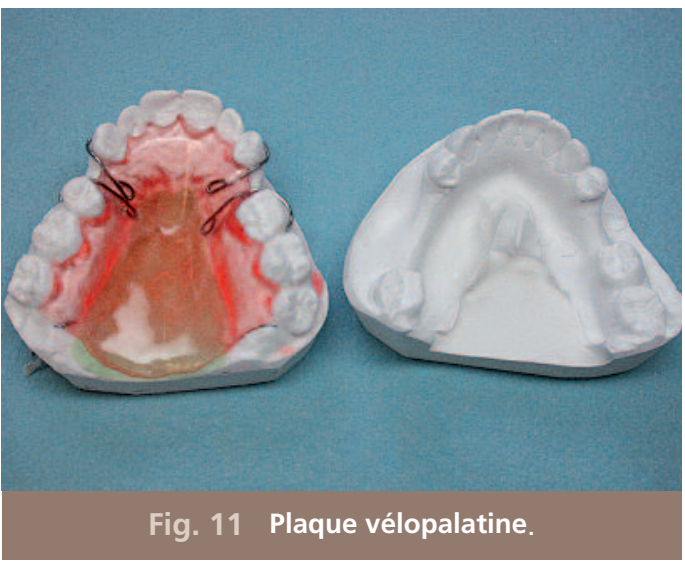

matériau souple, muqueux, dynamique. II est porté par le patient pendant quelques jours et retouché avant d'être transformé en utilisant une résine dure (fig. 11).

\section{> Les prothèses obturatrices}

Elles représentent une part essentielle du travail du prothésiste maxillo-facial d'un centre anticancéreux. Elles servent à combler une perte de substance maxillaire et palatine après exérèse chirurgicale et sont donc sur le même créneau que les techniques de reconstruction avec lesquelles elles ont été longtemps en compétition. Aujourd'hui, ces deux approches ont appris à collaborer pour permettre au patient de bénéficier des avantages de chacune d'entre elles. Elles ont un rôle fonctionnel essentiel puisqu'elles doivent permettre au patient de retrouver la mastication, une étanchéité entre cavités buccale et nasale et enfin l'élocution et la déglutition en corrigeant l'insuffisance vélaire.

\section{> Les prothèses plastiques faciales}

Elles sont appelées également épithèses. Elles permettent de masquer des mutilations faciales importantes telles que les amputations du nez ou du pavillon de l'oreille. Elles ont une fonction essentiellement esthétique, en étant au mieux invisibles, et parfois fonctionnelles. II en existe différents types:

- les prothèses nasales ;

- les prothèses du pavillon de l'oreille ;

- les prothèses occulo-palpébrales.

Avec différents moyens de fixation :

- colle à peau ;

- implants ;

- paire de lunette.

Leurs indications sont principalement pour pallier les insuffisances de la chirurgie reconstructrice, l'exemple le plus évident étant le globe oculaire, dont le remplacement fait appel systématiquement à un matériel synthétique. Mais elles sont également utilisées dans le cas où l'on cherche à éviter un geste chirurgical lorsque la qualité des tissus locaux est médiocre, que le risque de récidive tumoral est grand ou que l'état général du patient est mauvais. Enfin, elles sont de plus en plus utilisées en collaboration avec la chirurgie reconstructrice afin que le patient puisse bénéficier de l'ensemble des avantages de ces deux techniques.

D'une manière générale, toute perte de substance de la face peut à la fois être comblée et recréée par une épithèse. Encore une fois, le but n'est pas d'opposer reconstruction chirurgicale et remplacement prothétique mais d'utiliser l'ensemble des possibilités offertes par ces deux spécialités à l'avantage du patient. II n'y a donc plus confrontation mais collaboration. Les indications, les associations et le choix définitif devront être pris en analysant l'ensemble des paramètres présentés par chaque cas clinique. Les principaux à prendre en compte sont :

- les limites de chaque technique ;

- les possibilités techniques de l'équipe soignante ainsi que ses habitudes ;

- la lourdeur de la prise en charge ;

- l'état général et le désir du patient. 


\section{Les moyens de protection}

\section{> Les prothèses de curiethérapie}

Elles servent à isoler les tissus sains du rayonnement radioactif et évitent l'ostéoradionécrose. Ces appareils sont réalisés en résine et en plomb de $4 \mathrm{~mm}$ d'épaisseur et ajustés au modèle en plâtre. Ces appareils sont destinés à être mis en bouche (fig. 12 et 13).

\section{> Les masques de plomb}

Ils sont destinés à la radiothérapie. Une empreinte en alginate du visage est réalisée, le
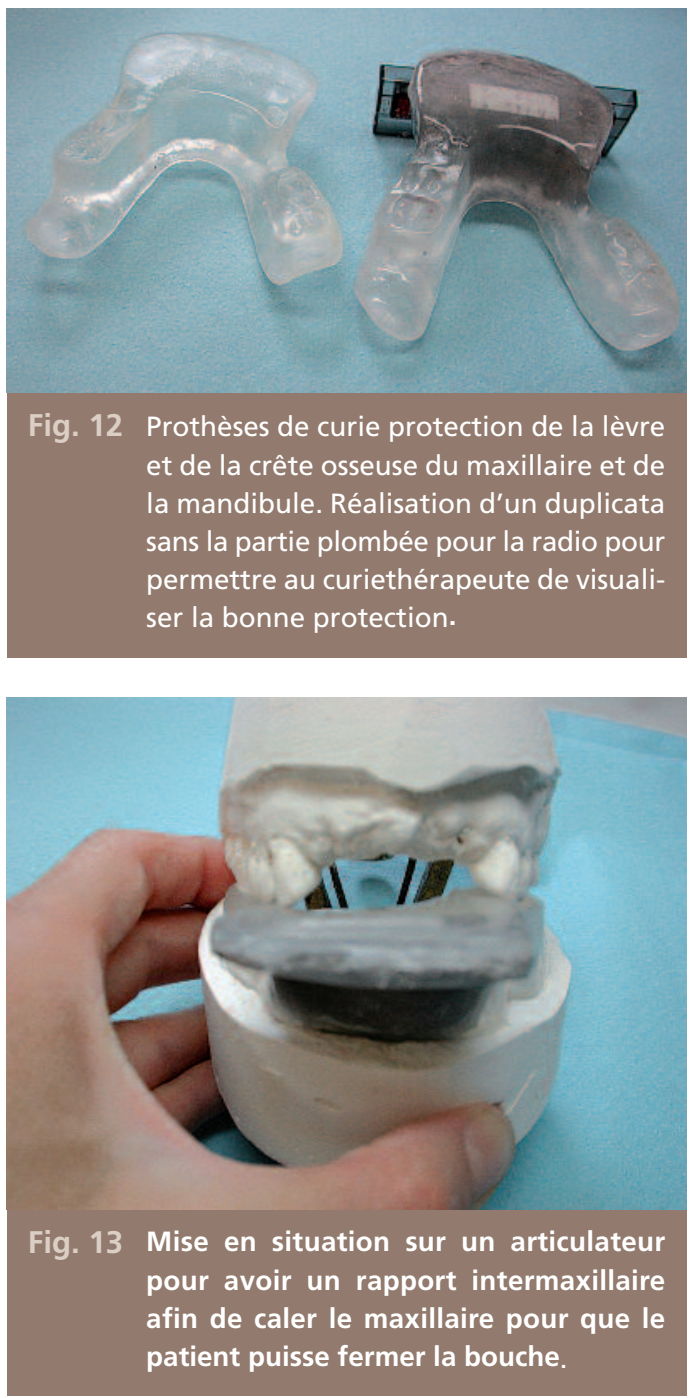

radiothérapeute nous indique la partie du visage qui va être irradiée, l'empreinte est coulée en plâtre extra-dur afin de façonner les plaques de plomb sur le modèle en plâtre, elle

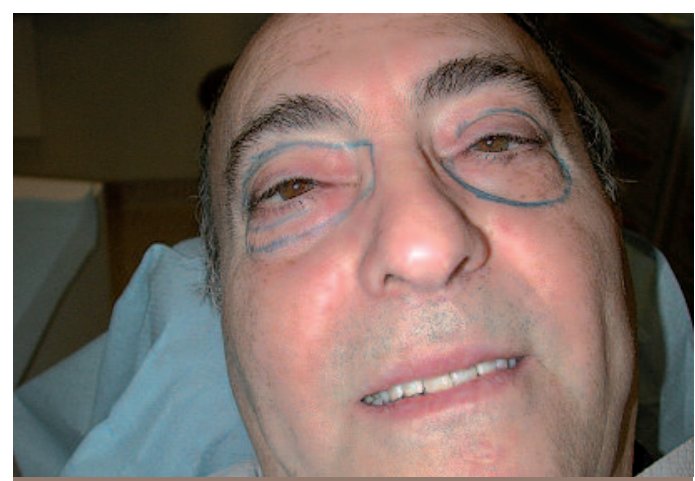

Fig. 14 Marquage par le radiothérapeute de la zone à irradier.
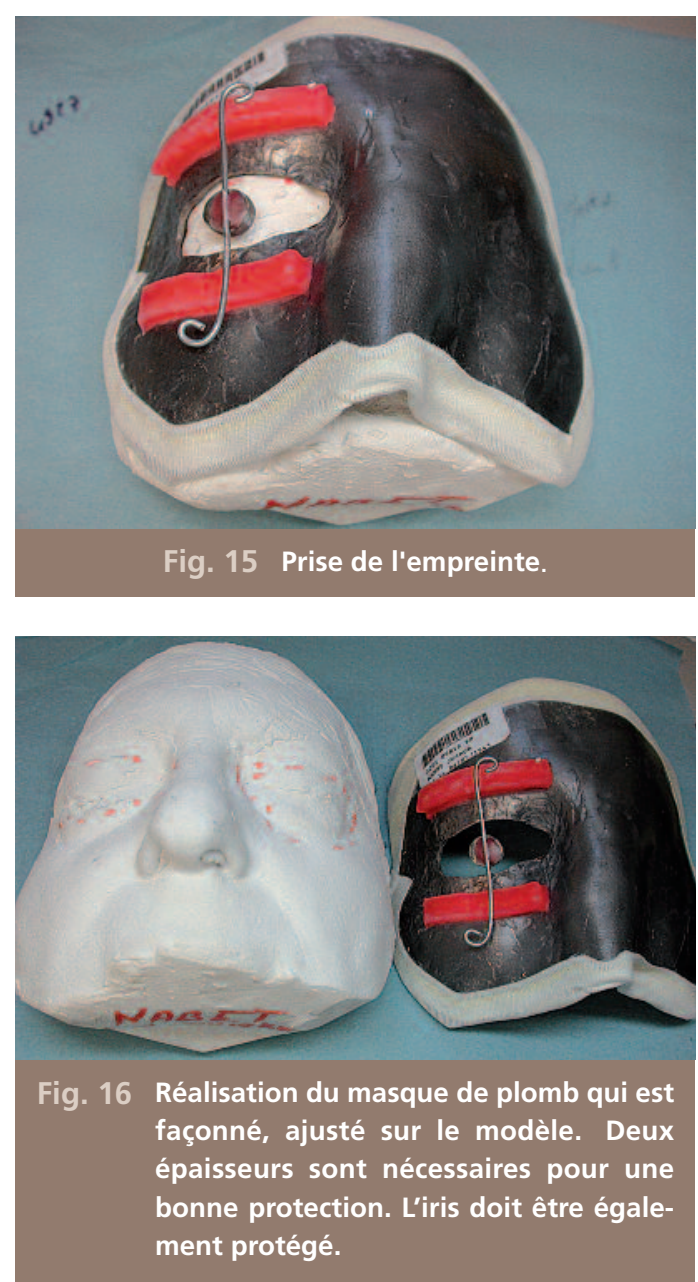
est faite en deux épaisseurs de $8 \mathrm{~mm}$. Ces appareils ont l'avantage d'être adaptés au traitement des tumeurs superficielles et évitent d'irradier les organes sous-jacents (fig. 14 à 16).

\section{Les moyens préventifs \\ au traitement \\ de la radiothérapie}

Ce sont les gouttières porte-gel fluoré (GPGF). Elles sont utilisées systématiquement par les patients pour la prévention des caries et de l'ostéoradionécrose à la suite d'un traitement de radiothérapie (fig. 17).

\section{Réalisation pratique d'une prothèse maxillo-faciale}

Pour pouvoir réaliser une prothèse maxillofaciale, le prothésiste doit partir de matériaux de base à l'état brut, de formes et de bases anatomiques et façonner à partir de ces matériaux une prothèse adaptée au modèle anatomique.

\section{> Les matériaux}

Plusieurs types de matériaux peuvent être utilisés. Les plus utilisés sont les suivants.

\section{- Les alginates}

Ce sont des hydrocolloïdes irréversibles qui servent à prendre des empreintes dentaires et faciales.

\section{- Les plâtres}

Il existe de nombreuses variétés de plâtres. Ils sont utilisés pour couler les modèles de travail.

On distingue :

- les plâtres de classe 2 , destinés à la réalisation des travaux courants et à la mise en moufle ;

- les plâtres de classe 3 , destinés aux prothèses mobiles et antagonistes ;

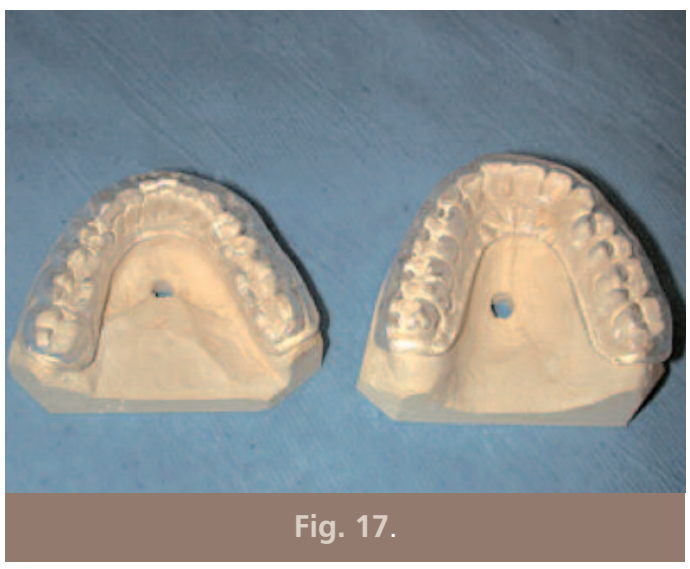

- les plâtres de classe 4, destinés aux prothèses fixes et châssis métallique. Ce plâtre a la particularité d'avoir une grande précision de par sa granulométrie très fine.

\section{- Les résines}

On retrouve ces matériaux en diverses consistances.

\section{- Les résines dures}

Elles sont utilisées pour la confection des prothèses maxillo-faciales. On en distingue deux types :

- les résines rigides sont constituées de résines acryliques à polymérisation lente. Elles sont couramment utilisées. Ce sont des résines thermoplastiques, elles se présentent sous la forme d'une poudre (polymère) et d'un liquide le (monomère). La polymérisation s'effectue à chaud sous forte pression avec une montée de température très lente. On obtient ainsi une résine non poreuse, moins cassante. En prothèse maxillo-faciale, on utilise souvent des résines transparentes sans colorant. Pour les prothèses obturatrices immédiates ou provisoires, cela permet de surveiller les tissus sousjacents ;

- les résines acryliques autopolymérisables: ces résines sont employées comme moyen temporaire de contention dans les fractures, comme 
matériaux de scellement pour la fixation des éléments rétentifs des endoprothèses ou des prothèses plastiques. Il en existe plusieurs variétés selon leurs utilisations. Leurs durcissements varient de 10 à 14 min selon la température ambiante. La texture reste poreuse, ce qui peut provoquer dans la cavité buccale des irritations du fait du résidu du monomère qui n'a pas été totalement polymérisé. Ces matériaux rendent service du point de vue de leur manipulation et du gain de temps.

\section{- Les résines souples}

Leurs principales qualités sont :

- une bonne résistance mécanique ;

- une résistance aux salissures ;

- une bonne stabilité de la teinte ;

- une résistance à la chaleur.

\section{- Les silicones}

Il existe trois catégories de produits à base de silicone :

\section{- les matériaux prêts à l'emploi}

Ils se présentent sous forme de plaque ou de préforme, ils sont utilisés en inclusions souscutanées pour corriger une dépression ou modifier un relief.

\section{- les gels de silicone}

Ils sont utilisés en prothèse maxillo-faciale pour remplir des poches préformées, elles-mêmes en silicone, ils sont destinés aux comblements des pertes de substance des parties molles.

\section{- les élastomères}

Ils sont utilisés en prothèse maxillo-faciale, ils se présentent sous la forme d'une pâte qui, lorsqu'elle est polymérisée, a une consistance élastique. Ils peuvent être fermes, durs ou souples. Grâce à leur perméabilité et à leur pouvoir antiadhérent, ils permettent au tissu dans lequel ils sont placés de respirer et de ne pas macérer. Ils présentent les qualités nécessaires pour réaliser des prothèses maxillo-faciales car ils résistent au vieillissement et aux salissures.

\section{Le modèle anatomique}

\section{> En pré-opératoire}

Le patient est informé de la limite de la résection qu'il va subir et des différentes possibilités de la restauration, des avantages et des inconvénients de la prothèse. Cette information permet au patient d'être préparé psychologiquement à cette épreuve.

Des empreintes primaires sont réalisées afin d'obtenir des modèles en plâtre. Le tracé et la limite de résection sont indiqués par le chirurgien. Les dents sont sectionnées sur le modèle en plâtre puis on réalise la future prothèse d'attente ou provisoire.

Dans le cas d'une radiothérapie, le prothésiste va confectionner des gouttières porte-gel fluoré (GPGF). A partir d'une plaque de silicone chauffée à température, elle sera estampée grâce à une machine de thermo-façonnage sur le modèle en plâtre.

Dans le cas d'une BPTM, une prothèse guide sera prévue. Celle-ci est réalisée à partir d'un modèle en plâtre. Des crochets sont façonnés sur les dents, la plaque et le volet sont réalisés en résine autopolymérisable. Le volet est mis du côté opposé à la résection.

Dans le cas d'une résection d'infrastructure avec reconstruction, plusieurs conformateurs vont être réalisés à partir de plusieurs moulages :

- le premier moulage nous sert de référence sur la forme et l'esthétique des dents ;

- le deuxième moulage nous sert à confectionner le patron, toutes les dents sont sectionnées afin de réaliser une plaque souple thermofaçonnée sur le modèle en plâtre. 
Celui-ci servira au chirurgien à modeler le lambeau ;

- le troisième moulage nous sert à préformer le futur lambeau sur le modèle en plâtre avec un Optosil. Les parties à réséquer sont indiquées par le chirurgien. Le conformateur est confectionné à partir d'une plaque souple thermofaçonnée sur le modèle en plâtre.

Les conformateurs narinaires sont réalisés en silicone. Ils sont préfabriqués au laboratoire, ont la forme de la narine, légèrement courbée et en lame de couteau.

Dans le cas d'une épithèse, avant le geste de résection, des empreintes de référence sont prises (quand c'est possible) afin de guider la réalisation de la future prothèse. II est nécessaire de prévoir les zones d'appui et de rétention de la prothèse, de déterminer l'emplacement des bords et des zones de jonction pour les dissimuler dans les sillons ou des plis natu-
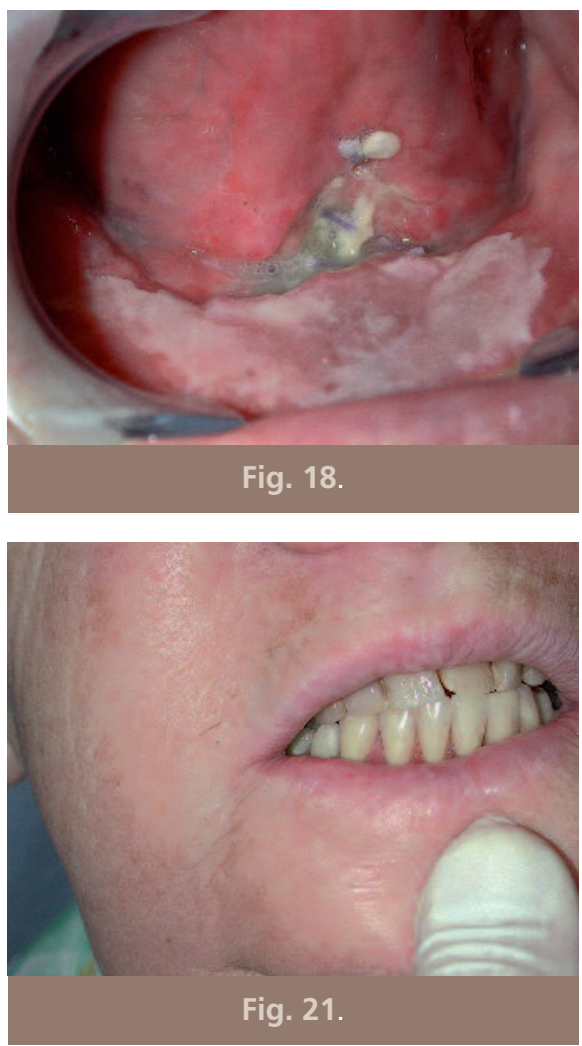
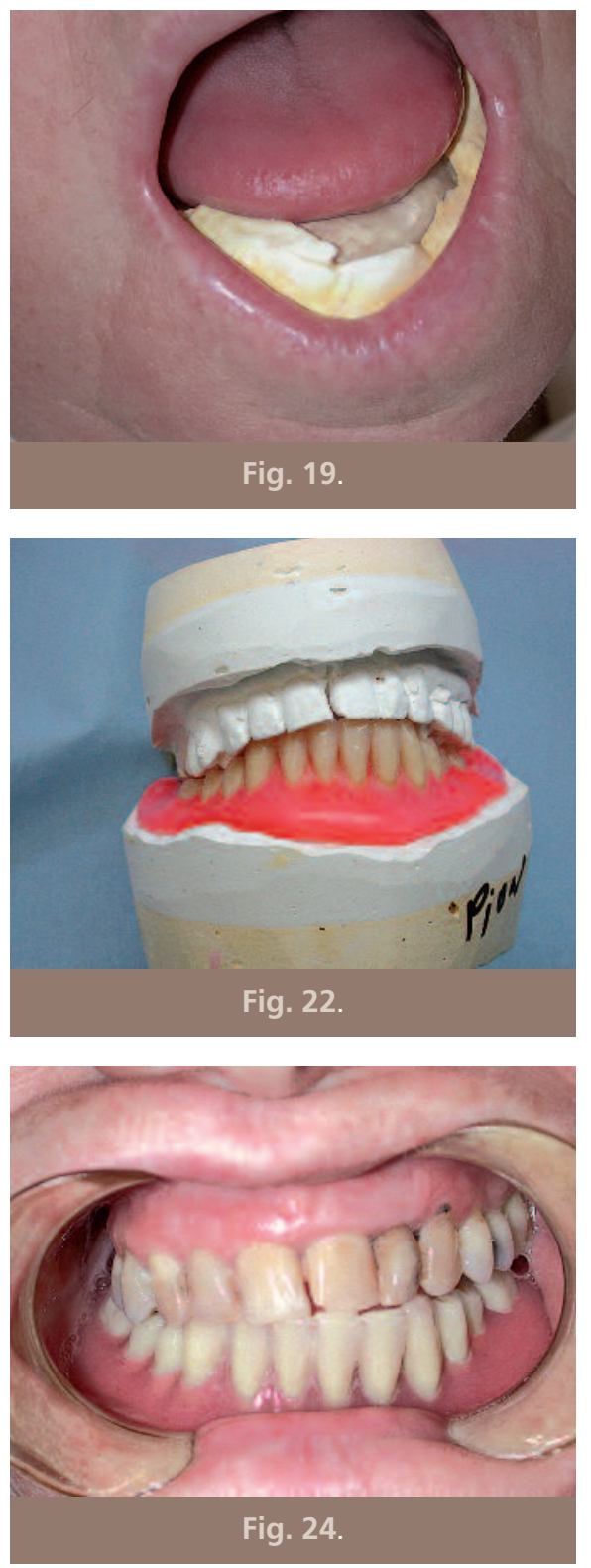
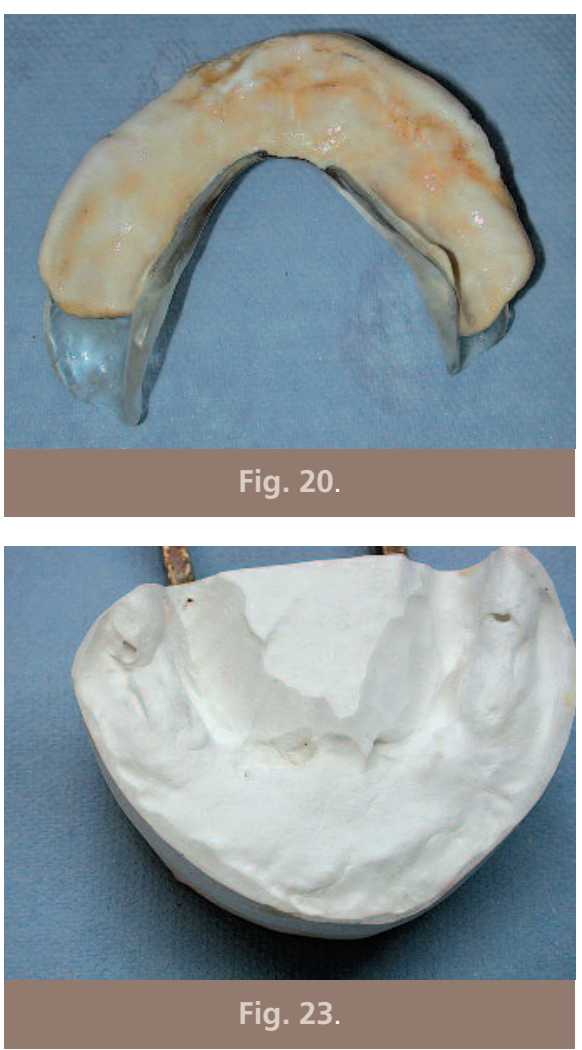
rels de la peau. Au moment de l'appareillage, le chirurgien peut être appelé à intervenir pour régulariser ou recouvrir le pourtour d'une perte de substance pour permettre une adaptation plus étanche de la prothèse.

\section{> En peropératoire}

Certaines empreintes sont difficiles à prendre pour des raisons de limitation d'ouverture buccale dues au volume tumoral mais aussi au caractère douloureux de la lésion. Dans ce cas, le prothésiste confectionne des conformateurs buccaux en résine dure préformée que le chirurgien va adapter grâce à un rebasage avec une base molle, dans le cas d'une libération de langue par exemple [2] (fig. 18 à 24).

Dans le cas d'une ablation sans reconstruction, le chirurgien va obstruer la cavité par un matériau en silicone dur. C'est à partir de cette balle obturatrice provisoire de référence que le pro- thésiste pourra confectionner la pièce définitive, ou réaliser dans certains cas un conformateur ou une plaque vélopharyngienne.

\section{> En postopératoire}

Le prothésiste participe aux suites postopératoires immédiates et à la prise en charge à long terme.

En postopératoire immédiat, le patient peut nécessiter des consultations itératives en odontologie afin d'adapter le conformateur aux variations locales secondaires à l'œdème et au phénomène de cicatrisation. C'est par exemple le cas des reconstructions de la voûte palatine par lambeau scapulo-dorsal où l'angle de la scapula, qui reconstruit le palais, est laissé en cicatrisation jusqu'à épithélialisation. Le prothésiste confectionne des conformateurs souples non traumatisants afin de guider la cicatrisation. Le patient est revu plusieurs fois afin de remodeler
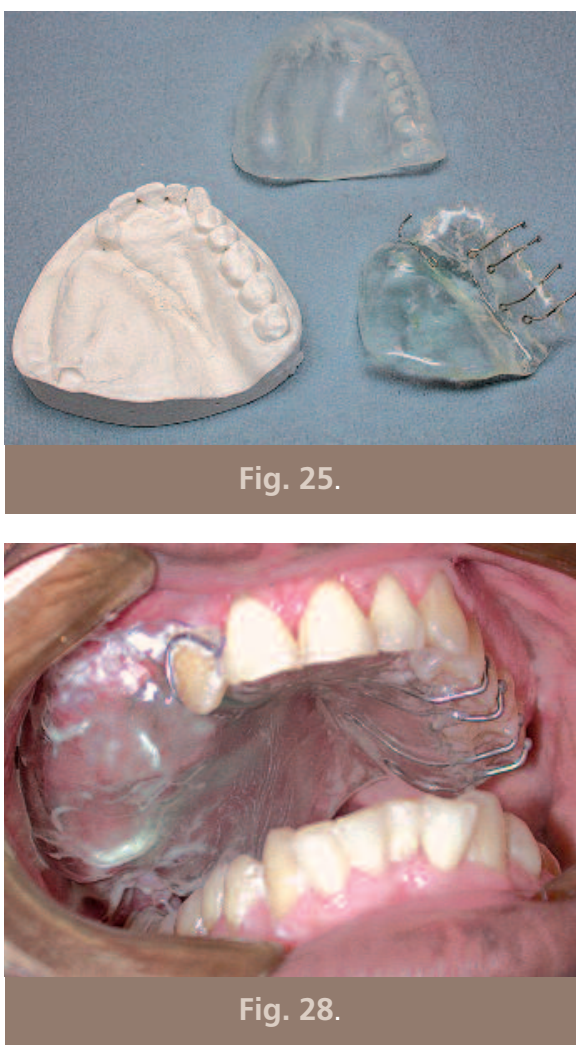
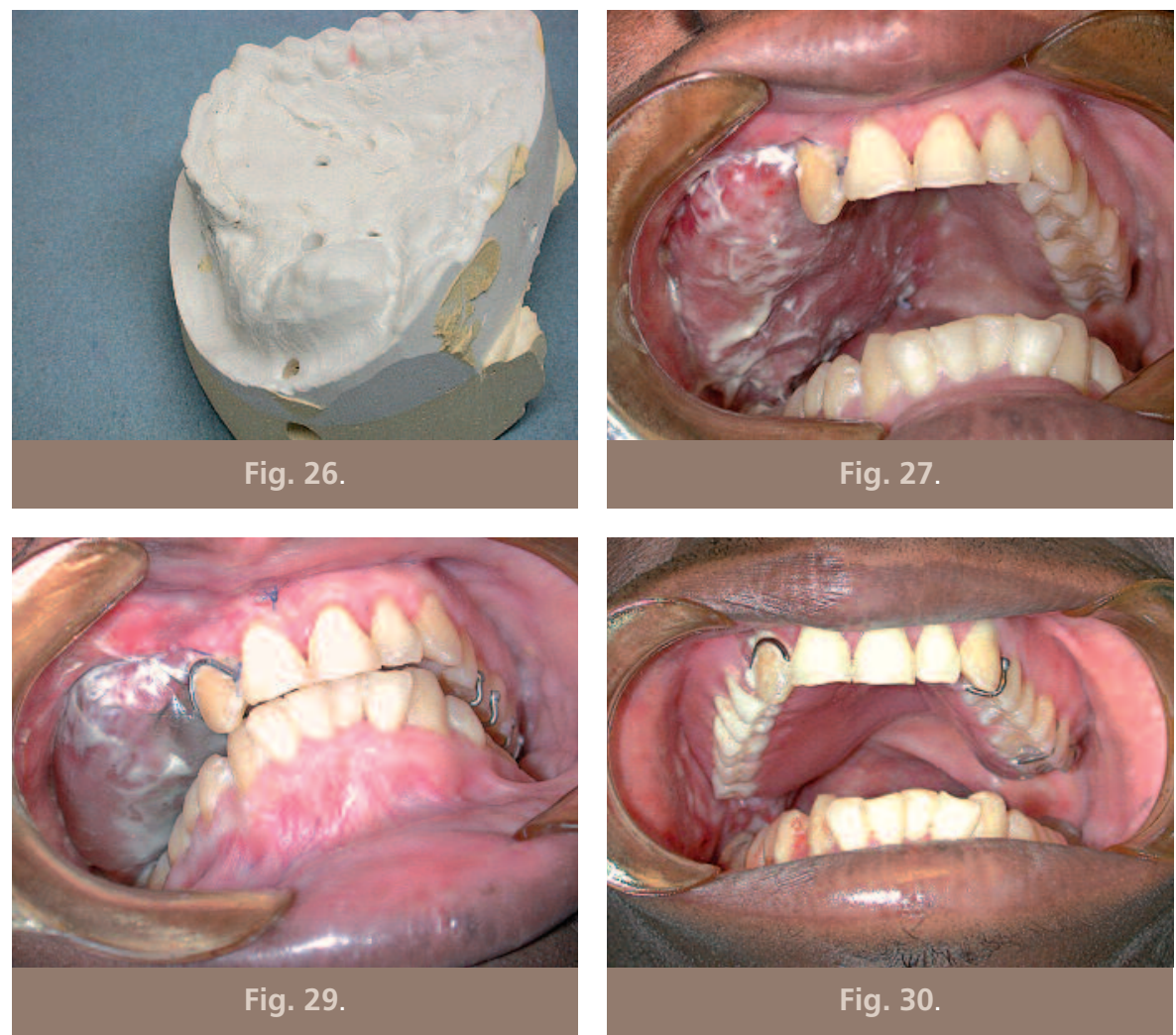
le modèle en plâtre, le conformateur est refait selon la nouvelle empreinte jusqu'à complète cicatrisation, ce qui permet de réaliser une réhabilitation définitive. Si le chirurgien prévient assez rapidement après l'intervention, on peut limiter ces rétractions cicatricielles, empêcher les brides de se former par un conformateur, assouplir certaines d'entre elles en voie de formation [5, 14] (fig. 25 à 30).

Afin d'éviter une constriction des mâchoires qui rendrait impossible tout appareillage ou toute intervention, on réalise une "sauterelle "», une sorte de mécanothérapie qui va aider le patient à travailler les muscles de sa mâchoire (fig. 31). À long terme, le prothésiste peut être amené à confectionner les différentes prothèses présentées plus haut, c'est-à-dire :

- les prothèses vélopalatines ;

- les prothèses guides ;

- les prothèses obturatrices ;

- les conformateurs ;

- les prothèses plastiques faciales ;

- les plaques de cicatrisation ;

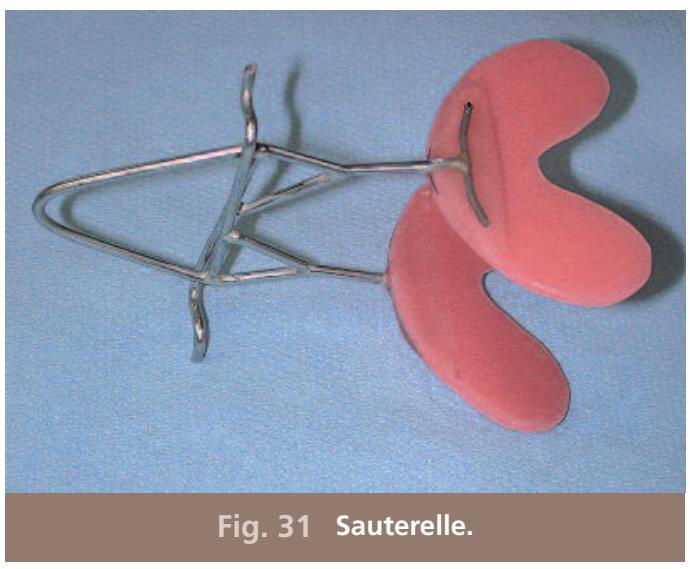

- les prothèses vissées ;

- les épithèses.

On rappelle que l'élaboration et la planification de tous ces appareillages commencent systématiquement dès la période préopératoire. II est en effet plus facile de préparer des appareils de maintien ou de soutien avant l'opération, même si ceux-ci sont approximatifs, car les modifications sont moins pénibles pour le patient.

Nous allons voir maintenant comment nos prothèses sont fabriquées.

\section{Méthode de fabrication des différents appareils}

\section{Introduction}

La perte de substance du maxillaire est dans la plupart des cas la conséquence de l'exérèse des tumeurs des VADS localisées au niveau de l'étage moyen de la face.

La chirurgie laisse des séquelles mutilantes tant au niveau anatomique, esthétique, psychologique que fonctionnel et seule une réhabilitation prothétique peut redonner au patient une fonction masticatrice, une phonation, une déglutition et un aspect esthétique satisfaisant et une intégration sociale [9-11].

\section{Les prothèses obturatrices}

Une prothèse obturatrice se compose de deux parties distinctes :

- une plaque palatine, qui restaure la surface du palais et dont la face inférieure se trouve 
en position intrabuccale. Elle renferme la portion dentée ;

- un obturateur qui est en position intramaxillaire afin de combler la perte de substance et qui vient reposer sur la face supérieure de la plaque.

L'obturateur souple est creusé et allégé au maximum. Il est amovible afin d'être remplacé dans le temps, car il est plus fragile qu'une résine. Ces indications le réservent aux patients édentés complètement dont la perte de substance s'étend à plus de la moitié de la voûte palatine.

L'obturateur rigide est utilisé chez les sujets dentés, dont la perte de substance est bien limitée.

\section{> Confection d'un obturateur rigide,} pour un patient denté, dans le cas d'un châssis métallique

\section{- Technique d'empreinte}

À partir du modèle en plâtre : confection d'un porte-empreinte individuel en résine photopolymérisable. Cette empreinte secondaire est coulée en plâtre dur. Le prothésiste maxillofacial va étudier cette empreinte secondaire afin de déterminer les limites de l'obturateur, ainsi que les replis muqueux cicatriciels, qui doivent être dégagés pour ne pas irriter. Une mise en dépouille de la cavité et du modèle va être effectuée grâce à un paralléliseur.

Le prothésiste maxillo-facial va dessiner la future plaque métallique.

Les collets des dents doivent être dégagés, mais tout en gardant une bonne surface d'appui sur la muqueuse. La plaque va se prolonger en avant de la perte de substance par une grille rétentive de façon qu'elle soit complètement noyée dans la résine.

À partir de ce schéma, le prothésiste va dupliquer ce modèle, qui sera réalisé en revêtement et qui sera durci au four à 150 degrés, afin que le modèle dupliqué ne soit pas friable lors de la réalisation de la maquette. La maquette est réalisée de la façon suivante :

- mise en cylindre,

- coulée avec un revêtement réfractaire à 1100 degrés, afin de faire fondre la cire,

- coulée du cylindre avec un alliage en nickelchrome,

- grattage, polissage.

- Étapes

- L’armature métallique est essayée.

- La réalisation d'une cire d'occlusion sur l'armature métallique est faite.

- La teinte des dents est prise.

- La mise en occlusion est effectuée sur l'articulateur.

- La dimension verticale est légèrement augmentée afin de compenser le tassement de l'appareil.

- Le montage des dents et l'essayage de la maquette s'effectuent.

- Une surempreinte de l'obturateur est effectuée afin d'obtenir une meilleure étanchéité.

- La maquette est mise en moufle. Toute la partie qui était en cire sera transformée en résine, puis cuite pendant plusieurs heures à 90 degrés.

- L'appareil est démouflé, l'obturateur est évidé afin d'alléger au maximum.

- Le bord de l'orifice est biseauté afin d'appliquer une cire calibrée pour fermer la zone évidée.

- Après transformation de cette cire en résine, elle sera scellée à l'obturateur grâce à une résine autopolymérisable.

- Grattage et polissage. 


\section{Prothèse obturatrice immédiate}

Elle est mise en place le jour de l'intervention et doit donc être préparée en préopératoire à partir des empreintes prises.

Elle ne comporte que la plaque palatine, puisque la portion obturatrice sera réalisée par l'opérateur après que l'exérèse soit finie.

Elle permet d'assurer une protection et une étanchéité immédiate et est utilisée pendant quelques semaines $[12,13]$.

\section{$>$ Chez le patient denté}

La plaque palatine peut inclure des crochets de contention destinés à sécuriser l'obturateur en Optosil.

\section{> Chez le patient édenté}

L'absence de dents interdit la réalisation d'une plaque palatine.

La solution de remplacement est de confectionner une prothèse palatine souple en silicone pour maintenir les mèches de tulle gras.

Un moule en alginate de la perte de substance est fait en peropératoire puis retiré.

Le patient sort de salle avec un pansement gras dans la cavité d'exérèse et le moule est adressé au prothésiste pour préparer pour le lendemain la balle obturatrice en silicone.

Un moule de l'empreinte est réalisé en plâtre dur et la technique de cire perdue est utilisée pour en obtenir un obturateur en silicone. $\mathrm{Ce}$ dernier est similaire à celui utilisé pour les épithèses. Il se présente sous forme d'une base et d'un catalyseur de couleur transparente.

L'ensemble est mis dans une cocotte sous pression afin de débuller le silicone. La prise durera 12 heures. Les bavures de silicone seront reti- rées au ciseau, la balle sera régularisée afin de ne pas blesser.

Ainsi, le lendemain de l'intervention, le patient peut disposer de la balle obturatrice.

\section{Prothèse obturatrice secondaire}

C'est une prothèse plus élaborée. Elle assure la mastication et l'obturation jusqu'à la cicatrisation complète des tissus.

\section{> Confection de la prothèse pour un patient denté}

La technique d'empreinte est la même que pour l'obturateur immédiat.

Le modèle de travail est réalisé, le fond de l'obturateur est comblé par du plâtre, les parois sont également mises en dépouille afin de préparer la plaque palatine munie de crochets et d'un prolongement qui pénètre dans la perte de substance.

L'articulé sera pris sur cette plaque rigide, qui est stable et qui va assurer un enregistrement précis de l'occlusion grâce à un articulateur qui permet de le mettre en face du moulage dentaire antagoniste.

Les dents peuvent être alors montées. Le montage est essayé. Il est modifié par la réalisation d'une surempreinte en élastomère afin d'adapter parfaitement l'obturateur à la perte de substance.

Ce montage est transformé en résine. Cet obturateur n'est cependant pas définitif car il devra être retouché en fonction des modifications de la perte de substance.

\section{> Confection de la prothèse pour un patient édenté}

La technique de réalisation est identique. La rétention de la prothèse est obtenue en utilisant toutes les parties saines. 
Sa sustentation est améliorée en mettant un protège-raphé médian en étain sur la voûte palatine afin de créer un vide.

\section{Prothèse obturatrice d'usage}

Si la plaque palatine est le plus souvent définitive, l'obturateur définitif est réalisé à distance lorsque les phénomènes de cicatrisation ont abouti à une perte de substance stable, non rétractile.
La prothèse définitive doit apporter au patient confort, fonction et esthétique, en s'approchant au maximum de l'état préopératoire.

La qualité de vie du patient en dépend.

Ainsi, elle doit permettre au patient de retrouver une mastication, une déglutition et une phonation proche de la normale, qui seules assurent au patient la possibilité de se réinsérer dans sa vie familiale, sa vie sociale et professionnelle [1-8] (fig. 32 à 40).
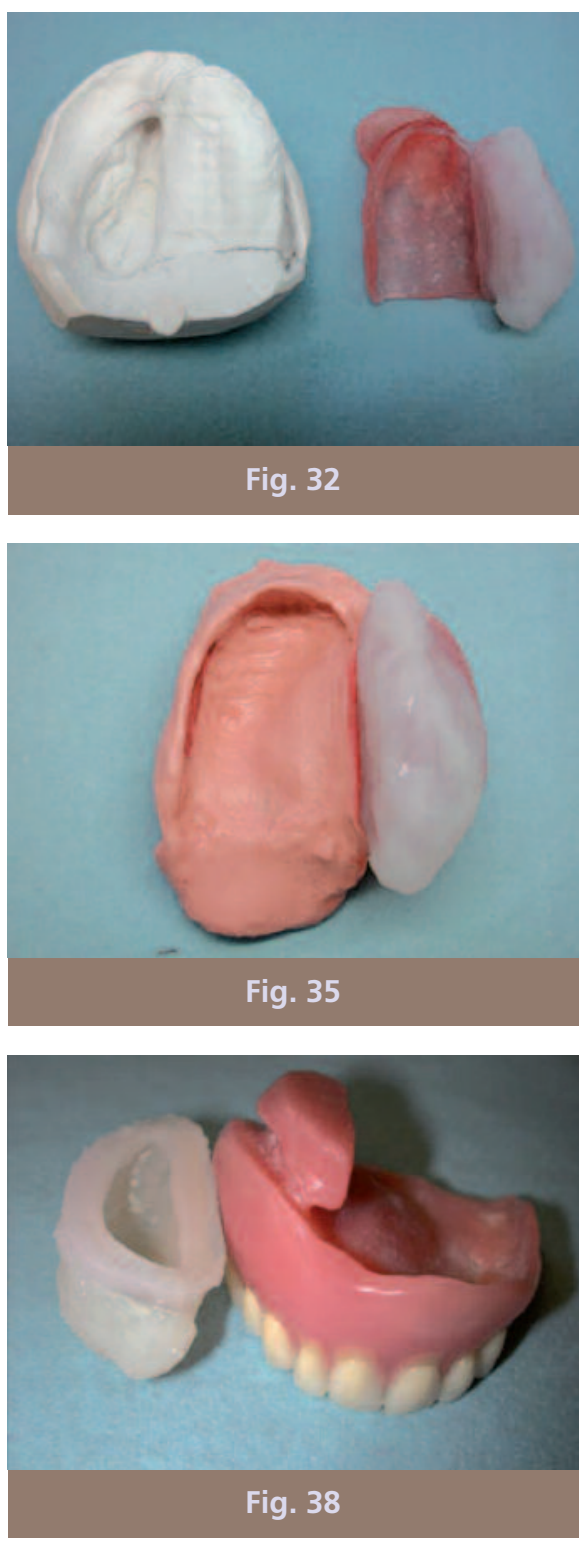
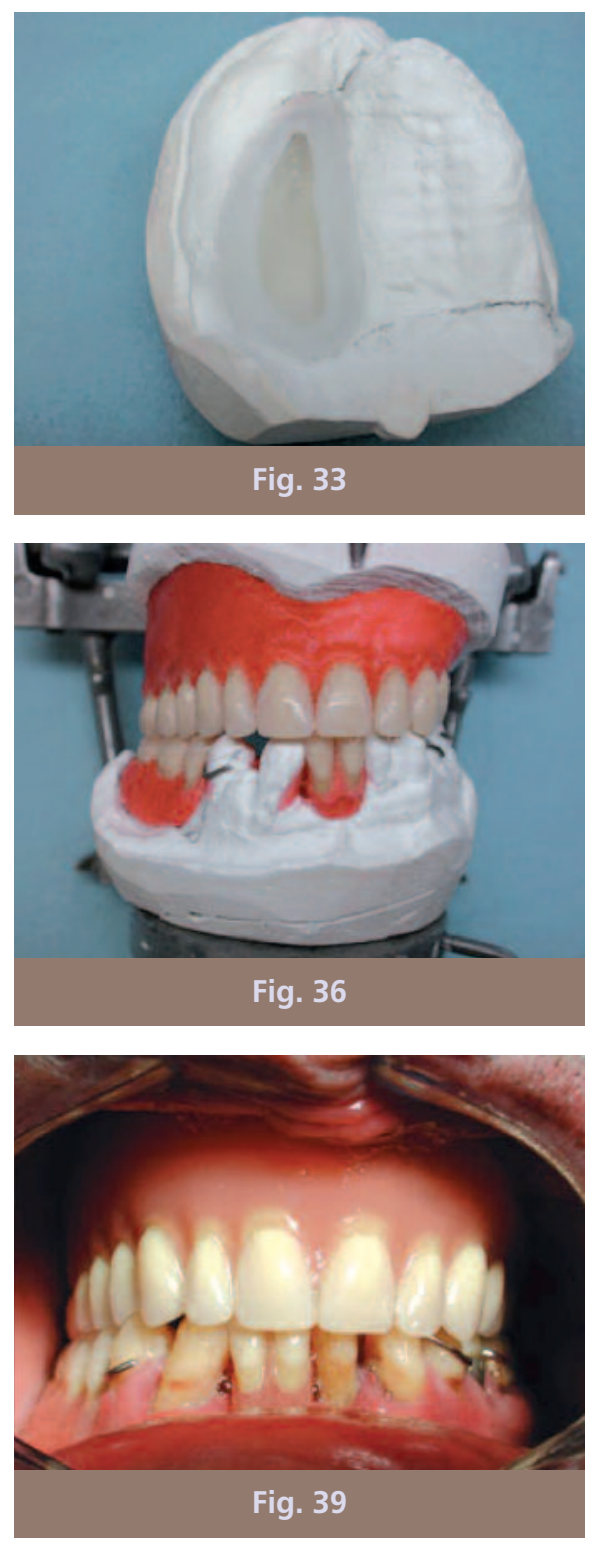
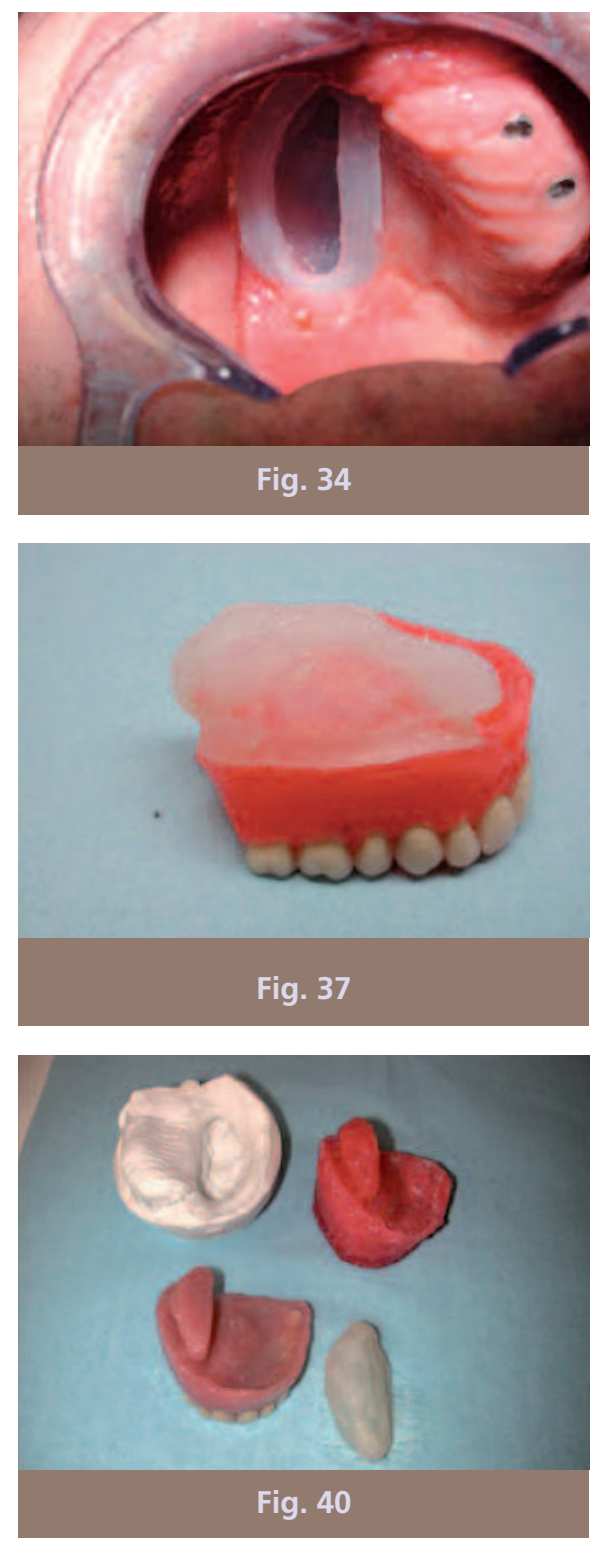


\section{Conclusion}

Dans le cas des prothèses obturatrices et pour pallier le problème d'étanchéité, la reconstruction par greffon microvascularisé associée à des implants dentaires est de plus en plus utilisée et contribue à un équilibre prothétique et fonctionnel et ceci grâce à l'imagerie médical en 3D. La prothèse et la reconstruction restent une complémentarité afin d'optimiser la prise en charge du patient. Une collaboration non seulement de toute l'équipe médicale et paramédicale mais aussi de celle du patient est essentielle dans la réussite de la réhabilitation fonctionnelle et esthétique.

\section{Bibliographie}

1. Bentahar $\mathrm{O}$, Pomar $\mathrm{P}$, Fusaro $S$, Benfdil $F$, Aguenaou A, Abdedine A. Prothèses obturatrices après maxillectomie : bases fondamentale et thérapeutiques. Encycl Méd Chir (Elsevier SAS, Paris) Stomatologie 2008;22-066-B-58, Médecine buccale 28-560-V-10.

2. Vigarios $E$, Pradines $M$, Fusaro S, Toulouse E, Pomar P.

Réhabilitation prothétique des pertes de substances mandibulaires d'origine carcinologique. Encycl Méd Chir (Elsevier SAS, Paris) 2007;22-066-B-51, Médecine buccale 2008;28-555-V-10

3. Saade AK, Caron J, Pomar P, Toulouse E, Poulain G.

La relation cabinet-laboratoire en prothèse maxillo-faciale. Real Clin 2002;13(2):191-201.

4. Barrelier P, Pilli $M$, Charles $B$, Lacacheux $B$, Compère JF. Appareilllage des malades après résection mandibulaire interruptrice. Rev Réhab Proth Maxillofac 1985;14:15-8.

5. Banzet P, Servant JM. Chirurgie plastique reconstructrice et esthétique.

$2^{\mathrm{e}}$ partie : tête et cou Paris : Flammarion, 1994.

6. Benoist M, Dichamp J, Poulain G.

Les problémes esthétiques de restauration prothétique de la face en fonction de l'environnement. Act Odonto Stomatol 1984;38(148):619-30.

7. Dichamp J, Guilbert F, Vaillant JM.

Prothèse plastique faciale..

Encycl Méd Chir

(Elsevier SAS, Paris)

Stomatologie 1990;22-087-M-10.

8. Pomar P, Pessey JJ, Soulet H. Prothèse obturatrice après maxillectomie.. Rev Laryngol Otol Rhinol 1994;115:147-51.

9. Thomas KF.

Prosthetic rehabilitation. London: Quintescence Publishing, 1994.
10. Toulouse $\mathrm{E}$, Pomar $\mathrm{P}$, Pradines M, Soulet $\mathrm{H}$. De la prothèse dentaire à la prothèse maxillo-facial : la prothèse obturatrice. Prothet Dent 1997;125:11-5.

11. Chelly H, Kadiri F, Laalou $Y$, Chekkoury L. La réhabilitation prothétique : une nécessité dans les cancers du maxillaire supérieur. Rev Maroc Odontol 1998;26:15-6.

12. Margainaud JP, Sarry B. Prothèses obturatrices après résection du massif facial : intérêt de la prothése immédiat. In: Jortay AM. (éd.). Rhéhabilitation anatomique et fonctionnelle après les traitements des cancers bucco-pharyngés. Paris : EDK, 1998:109-12.

13. Barrellier $P$, Feuillie $F$, Lecacheux B, Louis MY, Dao T.

Stratégie prothétique dans l'appareillage des pertes de substances maxillaires. Rev Réhab Proth Maxillofac 1988; 17:1-2,21-23 
14. Marandas $P$, Germain $M$, Margainaud JP, Hartl D, Kolb F. Chirurgie des tumeurs malignes

du plancher buccal :

exérèse et réparation.

Encycl Méd Chir
(Elsevier SAS, Paris)

Techniques chirurgicales,

Tête et cou

2006:46-240.

\section{A maxillo-facial prothetist within a cancer center}

Ilham EL ACHHAB

\section{Keywords \\ - former \\ - means of protection \\ - preventive methods \\ for treatment \\ - obturator prostheses}

The loss of secondary tissues in cancer resections of the cephalic extremity poses complex rehabilitation problems requiring an essential multidisciplinary approach among ENT surgeons, plastic surgeons, odontologists, radiotherapists, psychiatrists, speech therapists and prosthetists.

The goal is to offer patients the best combination of all these disciplines. Participation by the prosthetist is vital in order to address complex problems that no other discipline is able to take in hand.

Prosthetists contribute by providing prostheses and epitheses for various clinical situations that require a sense of creativity, esthetics and flexibility.

\section{Apprenez à respirer à vos enfants}

\section{Jean-Paul Allaux}

Les maladies ORL avec leur prolongement au niveau pulmonaire sont malheureusement de plus en plus répandues de nos jours et à tout âge.

La pollution atmosphérique, les appartements surchauffés ou enfumés par des parents tabagiques, les rues des villes saturées de gaz d'échappement... tous ces maux contemporains bien connus sont bien souvent responsables de l'installation de ces affections dès la petite enfance. Ils freinent par ailleurs le développement harmonieux du corps en installant parfois des déformations vertébrales mais aussi du palais et des mâchoires provoquant une mauvaise implantation des dents.

Fruit d'une longue expérience, ce livre se veut avant tout pratique. De nombreux exercices spécifiques à faire à la maison ou à l'école, seul ou en groupe, permettront aux éducateurs, parents et enseignants de trouver remède à ces différentes affections et d'aider efficacement les enfants à s'épanouir pleinement.

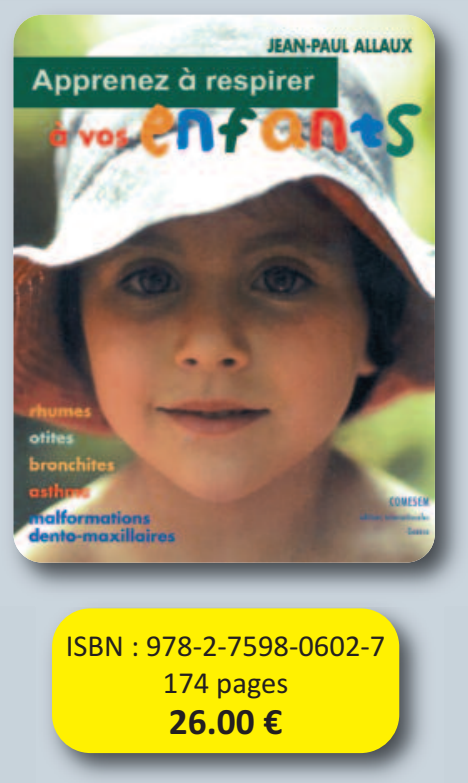

Un livre pour apprendre à respirer à nos enfants.

\section{Commandez directement sur : www.librairie-garanciere.com}

